\title{
Noninvasive Diagnosis of a Wide QRS Complex Tachycardia Mechanism by Programmed Atrial and Ventricular Stimulation Using a Dual Chamber Defibrillator
}

\author{
CHRISTIAN STICHERLING and GREGORY F. MICHAUD \\ From the Division of Cardiology, Department of Internal Medicine, University of Michigan \\ Medical Center, Ann Arbor, Michigan
}

\begin{abstract}
STICHERLING, C., ET AL.: Noninvasive Diagnosis of a Wide QRS Complex Tachycardia Mechanism by Programmed Atrial and Ventricular Stimulation Using a Dual Chamber Defibrillator. Stored atrial and ventricular electrograms retrieved from dual chamber implantable defibrillators facilitate the diagnosis of arrhythmias. This case also illustrates the usefulness of programmed atrial and ventricular stimulation for noninvasive rhythm diagnosis in patients with a wide QRS tachycardia and an implantable defibrillator. (PACE 2001; 24:1097-1099)
\end{abstract}

\section{dual chamber defibrillator, ventricular tachycardia, supraventricular tachycardia}

\section{Case Report}

An 85-year-old man with coronary artery disease and a left ventricular ejection fraction of 0.25 was admitted to the hospital for frequent shocks from his implantable defibrillator secondary to a wide QRS complex tachycardia at a rate of 124 beats/min (Fig. 1). He received an implantable dual chamber defibrillator 6 months prior to the admission for sustained ventricular tachycardia (VT). The 12-lead electrocardiogram (ECG) in sinus rhythm showed a left bundle branch block (Fig. 2). The differential diagnosis for this regular, wide QRS complex tachycardia, therefore, includes septal VT, bundle branch reentrant tachycardia, or a supraventricular tachycardia (SVT) such as atrioventricular nodal reentrant tachycardia (AVNRT), orthodromic reciprocating tachycardia (ORT), atrial tachycardia, atrial flutter, or SVT using an atriofascicular pathway.

Interrogation of the implantable defibrillator showed numerous episodes that were predominantly terminated by antitachycardia pacing and occasionally by a shock. The tachycardia was reproducibly induced by programmed atrial stimulation by the defibrillator. Atrial and ventricular

Supported in part by a grant from the German Research Foundation (DFG).

Address for reprints: Christian Sticherling, M.D., Division of Cardiology, Department of Internal Medicine, University of Michigan Medical Center, 1500 East Medical Center Drive, Box 0022, Ann Arbor, MI 49109-0022. Fax: (734) 936-7026; e-mail: csticherling@yahoo.com

Received June 26, 2000; revised September 26, 2000; accepted October 10, 2000. electrograms were recorded and ventricular pacing was performed at a cycle length slightly shorter than the tachycardia cycle length (Fig. 3).

\section{Commentary}

This case illustrates the usefulness of atrial electrograms and programmed atrial and ventricular stimulation for rhythm diagnosis in patients with implantable defibrillators. Although the most likely origin of a regular wide QRS complex tachycardia in a patient with coronary artery disease and depressed left ventricular ejection fraction is ventricular, ${ }^{1}$ one must be cautious not to miss other diagnoses that are amenable to curative treatment.

Analysis of the wide QRS complex tachycardia on the 12-lead ECG demonstrated an RS complex and an $\mathrm{R}$ to $\mathrm{S}$ interval $<100 \mathrm{~ms}$ in all precordial leads, and there was no evidence of atrioventricular (AV) dissociation and morphology criteria in $\mathrm{V}_{1}$ or $\mathrm{V}_{6}$ favoring SVT. ${ }^{2}$ Furthermore, the QRS morphology during tachycardia resembled the left bundle branch block during normal sinus rhythm.

The presence of 1:1 AV association as observed in the atrial and ventricular electrograms stored by the implantable cardioverter defibrillator (ICD) is additional evidence against VT. Two pacing maneuvers performed through the dual chamber defibrillator also strongly suggested the diagnosis of a SVT and established the probable tachycardia mechanism. First, atrial premature extrastimuli reproducibly induced the tachycardia following a sudden prolongation of the $\mathrm{AV}$ conduction time by $>50 \mathrm{~ms}$. This observation is characteristic of dual AV nodal physiology and 

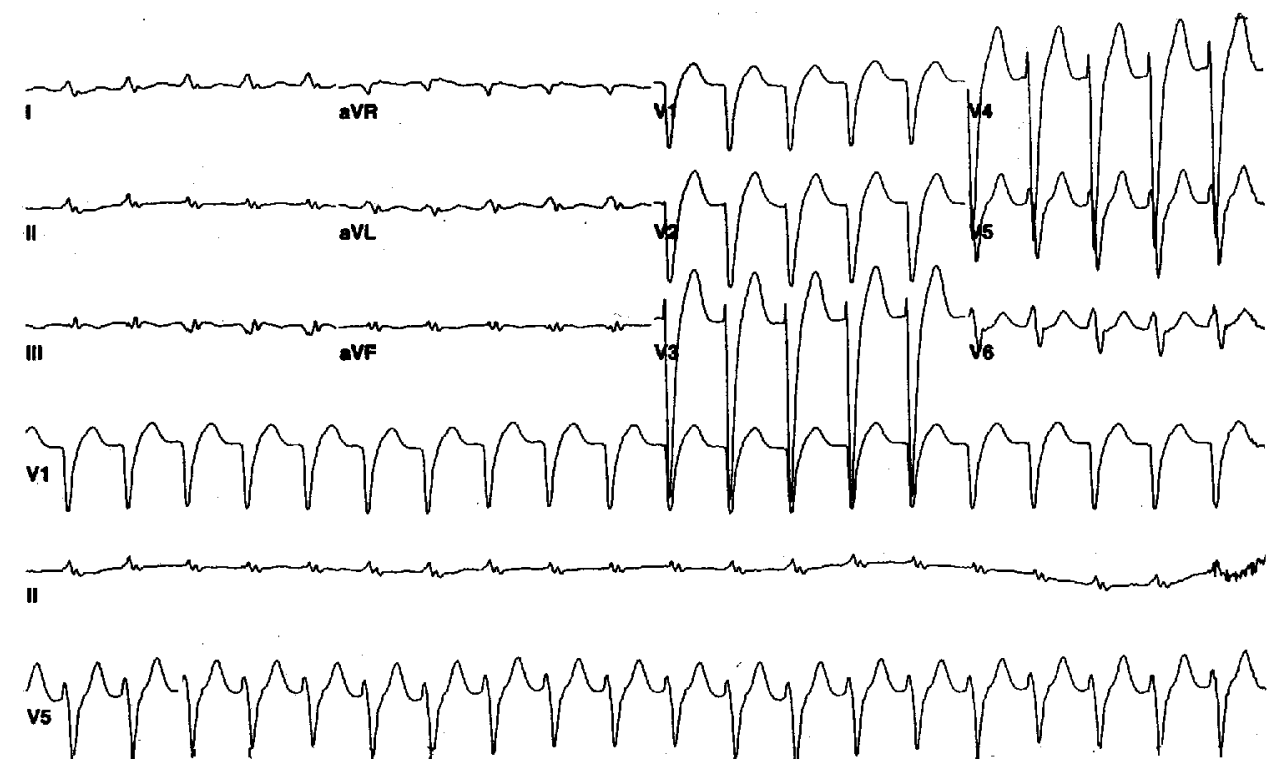

Figure 1. Twelve-lead electrocardiogram (ECG) of a wide-QRS complex tachycardia (paper speed $25 \mathrm{~mm} / \mathrm{s}$ ).
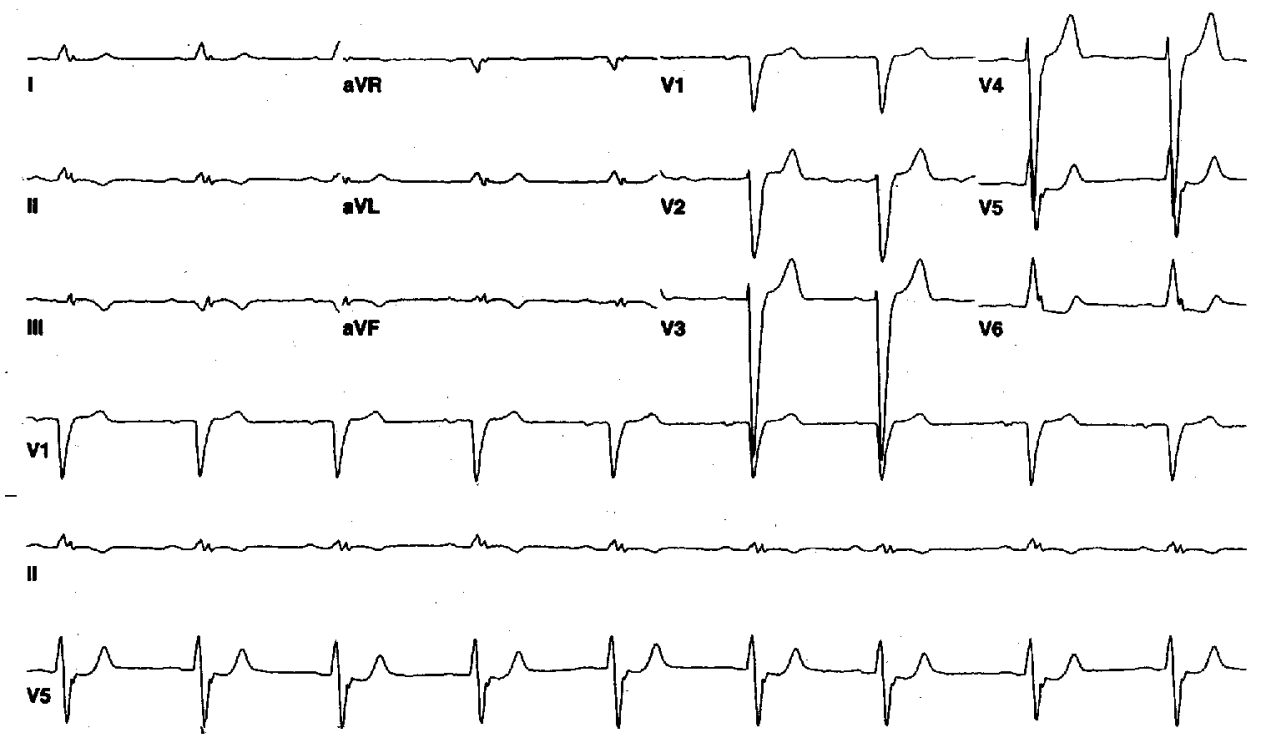

Figure 2. Twelve-lead electrocardiogram (ECG) during sinus rhythm (paper speed $25 \mathrm{~mm} / \mathrm{s}$ ).

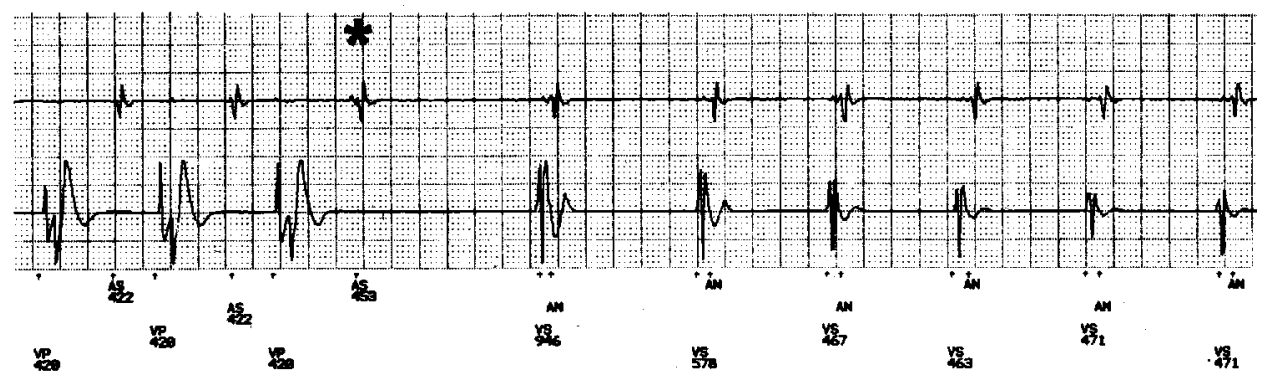

Figure 3. Intracardiac atrial (A) electrograms, ventricular (V) electrograms, and event marker during tachycardia. Ventricular pacing results in atrial entrainment and subsequent resumption of the tachycardia circuit. $V P=$ ventricular pacing; $V S=$ ventricular sensing; $A S=$ atrial sensing; $A N=$ "atrial noise," denoting atrial sensing in the postventricular atrial blanking period; numbers represent cycle lengths in milliseconds. 
strongly favors AVNRT, but does not completely exclude VT, ORT, or atrial tachycardia.

Second, entrainment of the tachycardia from the right ventricle at a cycle length just shorter than the tachycardia cycle length is a helpful diagnostic maneuver to distinguish paroxysmal SVTs. ${ }^{3}$ Figure 3 demonstrates that the last entrained atrial electrogram $\left({ }^{*}\right)$ is followed by the ventricular electrogram of the first tachycardia return cycle. Since the electrogram sequence immediately after the last paced ventricular electrogram shows an "atrial-ventricular" (AV) response, the diagnosis of an atrial tachycardia is extremely unlikely. Additionally, the intracardiac electrograms during tachycardia show a short ventriculoatrial

\section{References}

1. Baerman JM, Morady F, DiCarlo LA, et al. Differentiation of ventricular tachycardia from supraventricular tachycardia with aberration: Value of the clinical history. Ann Emerg Med 1987; 16: 40-43.

2. Brugada P, Brugada J, Mont L, et al. A new approach to the differential diagnosis of a regular tachycardia with a wide QRS complex. Circulation 1991; 83: 1649-1659. interval of $60 \mathrm{~ms}$, making ORT unlikely. ${ }^{4}$ The evidence suggested that typical AVNRT was the probable mechanism of tachycardia. A subsequent intracardiac electrophysiological study confirmed the diagnosis and radiofrequency ablation of the slow pathway ablation successfully abolished the tachycardia.

In summary, this case demonstrates the usefulness of dual chamber compared to single chamber implanted defibrillators for tachycardia diagnosis. Intracardiac atrial electrograms recorded during tachycardia and the ability to perform pacing maneuvers using the atrial and the ventricular leads provide additional valuable information for the correct arrhythmia diagnosis.

3. Knight BP, Zivin A, Souza J, et al. A technique for the rapid diagnosis of atrial tachycardia in the electrophysiology laboratory. J Am Coll Cardiol 1999; 33: 775-781.

4. Knight BP, Ebinger M, Oral H, et al. Diagnostic value of tachycardia features and pacing maneuvers during paroxysmal supreventricular tachycardia. J Am Coll Cardiol 2000; 36: 574-582. 